Supporting information for

\title{
Deep Profiling of Aminophospholipids Reveals a Dysregulated Desaturation Pattern in Breast Cancer Cell Lines
}

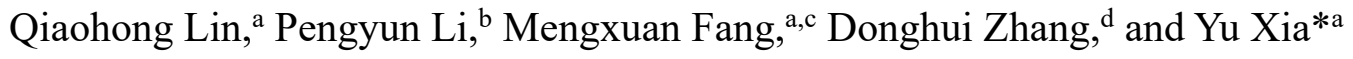 \\ aDepartment of Chemistry, MOE Key Laboratory of Bioorganic Phosphorus Chemistry \& \\ Chemical Biology, Tsinghua University, Beijing 10084, China

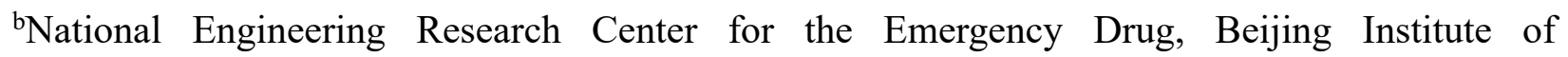 \\ Pharmacology and Toxicology, Beijing 100850, China

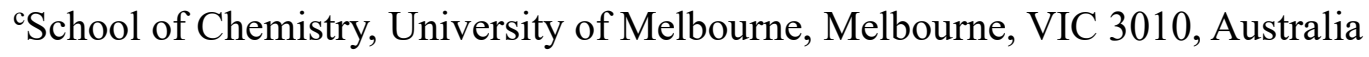 \\ ${ }^{\mathrm{d} D e p a r t m e n t ~ o f ~ P r e c i s i o n ~ I n s t r u m e n t, ~ T s i n g h u a ~ U n i v e r s i t y, ~ B e i j i n g ~ 10084, ~ C h i n a ~}$
}

\section{Table of Contents}

1. Chemical Reagents and Solvents...................................................................

2. Synthesis of TMAB-NHS Reagent.....................................................................

3. Cell Cultivation and Lipid Extraction......................................................................

4. RNA Extraction and Quantitative RT-qPCR ................................................

5. HILIC Separation Conditions and MS Parameters..................................................S-4

6. Pairing TMAB-NHS Derivatization with HILIC-PB-MS/MS .........................................S-5

7. Analysis of Aminophospholipids from Porcine Brain Lipid Extracts... .............................S-13

To whom correspondence should be addressed:

Prof. Yu Xia, xiayu@mail.tsinghua.edu.cn 


\section{Chemical Reagents and Solvents}

4-Trimethylammoniumbutyryl acid chloride, NHS, dicyclohexylcarbodiimide (DCC), anhydrous tetrahydrofuran (THF), triethylamine (TEA), $N, N$-dimethylformamide (DMF) were purchased from Beijing Innochem Science \& Technology Co., Ltd. (Beijing, China). HPLC grade chloroform $\left(\mathrm{CHCl}_{3}\right)$ was obtained from Beijing Tongguang Fine Chemicals Co. (Beijing, China). HPLC grade acetone, acetonitrile $(\mathrm{ACN})$, methanol $(\mathrm{MeOH})$, isopropanol (IPA), water, and acetic acid were purchased from Fisher Scientific (Pittsburgh, PA, USA). Ammonium acetate was purchased from Sigma-Aldrich (St Louis, MO, USA).

\section{Synthesis of TMAB-NHS Reagent}

4-Trimethylammoniumbutyryl acid chloride (1 g, $5.5 \mathrm{mmol})$ was dissolved in $125 \mathrm{~mL}$ of anhydrous acetonitrile in an ice bath. While stirring, 1.1 equivalent amount of NHS (0.695 g, 6.04 mmol) was added, followed by DCC $(2.15 \mathrm{~g}, 10.45 \mathrm{mmol})$. The mixture was stirred overnight at room temperature (over $12 \mathrm{~h}$ ). The reaction was monitored by TLC and the formed solid (i.e. 1,3 dicyclohexylurea) was filtered off. After solvent evaporation under vacuum, the crude residue was slurried with $62.5 \mathrm{~mL}$ of THF in an ice bath for $1 \mathrm{~h}$. The desired white precipitate was isolated, washed, and then dried under vacuum to give $1.1 \mathrm{~g}$ (4.5 mmol, $82 \%$ yield) final product. MS (Figure S1) were performed to confirm the structure of TMAB-NHS. The product was stored in the dark desiccator until further use.

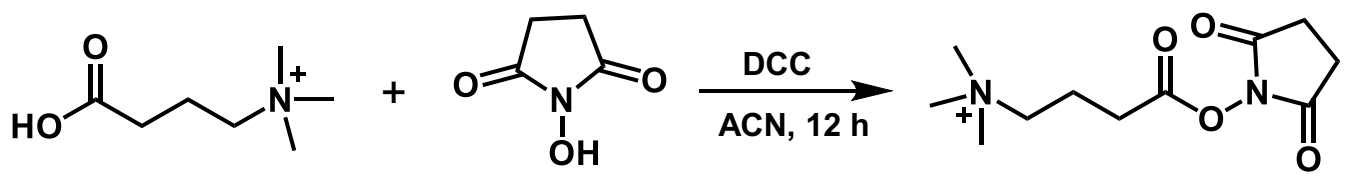

TMAB-NHS

Scheme S1. The synthetic procedure for TMAB-NHS reagent. 


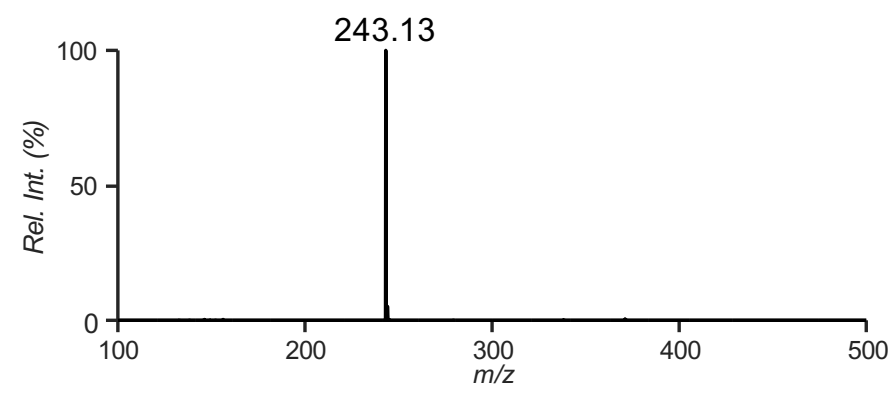

Figure S1. Positive-ion $\mathrm{MS}^{1}$ spectrum of TMAB-NHS $\left([\mathrm{M}+\mathrm{H}]^{+}\right)$reagent.

\section{Cell Cultivation and Lipid Extraction}

MDA-MB-231 cells were routinely cultured in Dulbecco's modified Eagle's medium (DMEM) containing high glucose (Invitrogen, Carlsbad, CA, USA). HMEC cells were cultured in HuMEC Ready Medium (ThermoFisher Scientific, Waltham, MA, USA). All of them were supplemented with 10\% fetal bovine serum (FBS) (Hyclone Laboratories Inc., Logan, UT, USA) and 1\% penicillin-streptomycin (Invitrogen) at $37{ }^{\circ} \mathrm{C}$ in a humidified incubator with $5 \% \mathrm{CO}_{2}$. After reaching $80-90 \%$ confluence, cells (approximately $3 \times 10^{6}$ ) were washed with ice-cold phosphate buffered saline (PBS) (Hyclone) and detached using 0.25\% trypsin solution (Hyclone). Cells were then resuspended in growth media and centrifuged at $5000 \mathrm{rpm}$ for $5 \mathrm{~min}$. After aspirating the media, cell pellets were washed twice with ice-cold PBS and lysed with $1 \mathrm{~mL}$ of MeOH. For lipid extraction, $2 \mathrm{~mL}$ of $\mathrm{CHCl}_{3}$ and $1 \mathrm{~mL}$ of water were added to the cell sample. The mixture was vortexed for $15 \mathrm{~min}$ and then was centrifuged at 10,000 rpm for $10 \mathrm{~min}$ to obtain layer separation. The bottom $\mathrm{CHCl}_{3}$ layer was collected and the extraction procedure was repeated by adding an additional $2 \mathrm{~mL}$ of $\mathrm{CHCl}_{3}$. The $\mathrm{CHCl}_{3}$ fractions were combined and dried under a stream of nitrogen. The extract was reconstituted in $1 \mathrm{~mL}$ of $\mathrm{MeOH}$ and stored at $-20{ }^{\circ} \mathrm{C}$ before analysis. For FADS2 inhibition, MDA-MB-231 cells was treated with $60 \mu \mathrm{M}$ FADS2 inhibitor (SC26196, purchased from Sigma-Aldrich, St Louis, MO, USA) for 72 hours, while the control group was treated with DMSO. 


\section{RNA Extraction and Quantitative RT-qPCR}

RNA was extracted from growing cells using TRIzol Reagent according to the manufacturer's instructions (Invitrogen, CA, USA). Cell sample after trypsin treatment was homogenized with TRIzol Reagent, mixed with $200 \mu \mathrm{L} \mathrm{CHCl}_{3}$ and vortexed for $1 \mathrm{~min}$, and then centrifuged for 15 $\min$ at $4{ }^{\circ} \mathrm{C}$. The upper aqueous phase was precipitated with $400 \mu \mathrm{L} \mathrm{IPA}$ for $10 \mathrm{~min}$, and then centrifuged for $10 \mathrm{~min}$. The acquired RNA pellets were washed once with $75 \%$ ethanol and $100 \%$ ethanol, respectively, and then air-dried, and dissolved in $100 \mu \mathrm{L}$ nuclease-free water. $2 \mu \mathrm{g}$ of RNA was used to prepare cDNA with oligo (dT) primers using PrimeScript ${ }^{\mathrm{TM}} \mathrm{RT}$ Master Mix (Takara, Shiga, Japan) in a total volume of $40 \mu \mathrm{L}$. qPCR was performed in triplicates in a $20 \mu \mathrm{L}$ SYBR Premix Ex Taq Master Mix (1×) (Takara, Shiga, Japan) containing $0.5 \mu \mathrm{M}$ of each of the primers and $10 \mathrm{ng}$ cDNA. Determination of relative mRNA levels was calculated using the comparative $\mathrm{Ct}$ method. $\beta$-actin was used for normalization.

\section{HILIC Separation Conditions and MS Parameters}

HILIC separation of lipids was performed using the following parameters. The gradient started from an initial ramp from $90 \% \mathrm{~B}$ to $85 \% \mathrm{~B}$ at $5 \mathrm{~min}$, held at $85 \% \mathrm{~B}$ within $5-8 \mathrm{~min}$, then decreased to $80 \% \mathrm{~B}$ at $15 \mathrm{~min}$, was followed by $70 \% \mathrm{~B}$ at $16 \mathrm{~min}$ and held at this composition for $4 \mathrm{~min}$, and returned to $90 \% \mathrm{~B}$ at $21 \mathrm{~min}$ and equilibrated for $5 \mathrm{~min}$ at flow rate of $200 \mu \mathrm{L} / \mathrm{min}$. The column was maintained at $35^{\circ} \mathrm{C}$. The injection volume was $2 \mu \mathrm{L}$.

The parameters of QTRAP 4500 were set as follows: ESI voltage, $\pm 4500 \mathrm{~V}$; curtain gas, $30 \mathrm{psi}$; interface heater temperature, $450{ }^{\circ} \mathrm{C}$; ion source gas 1 and gas 2, $30 \mathrm{psi}$; declustering potential, $\pm 100 \mathrm{~V}$. Data collection modes of enhanced MS (EMS), enhanced product ion (EPI) and $\mathrm{MS}^{3} \mathrm{CID}$ were used. Low resolution of Q1 was tuned (2 Da width), and collision energy at $15 \mathrm{eV}$ as well as high CAD gas were set for ion-trap CID.

X500R QTOF was used in positive-ion TOF MS mode and the optimized parameters were as follows: spray voltage, $5500 \mathrm{~V}$; curtain gas, $25 \mathrm{psi}$; CAD gas, 7 psi; ion source gas 1 and gas 2, 55 psi; interface heater temperature, $500{ }^{\circ} \mathrm{C}$; declustering potential, $100 \mathrm{~V}$. 


\section{Pairing TMAB-NHS Derivatization with HILIC-PB-MS/MS}

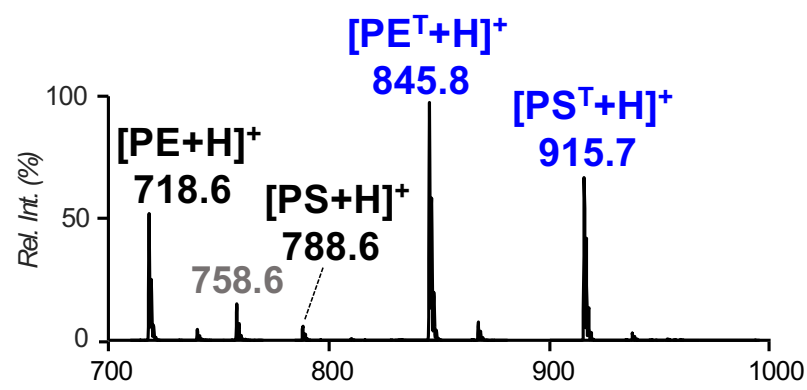

Figure S2. Positive ion HILIC-MS ${ }^{1}$ spectra of PE 16:0/18:1(9Z) and PS 18:1(9Z)/18:1(9Z) before (black trace) and after (blue trace) TMAB-NHS derivatization. The peak labeled with gray color represents Schiff base products. 
(a) Radicals generated from Norrish Type I cleavage of acetone

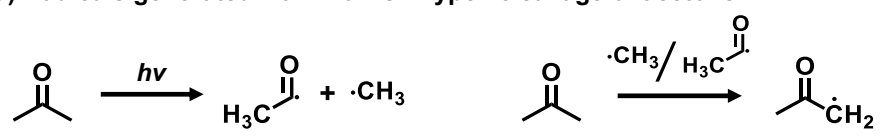

(b) Radical substitution on the acyl chain

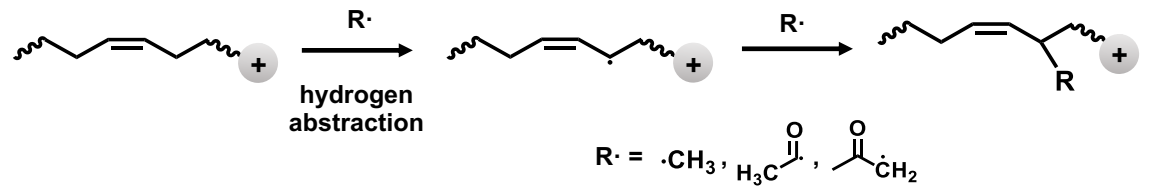

(c) Side products observed for plasmenyl-lipids

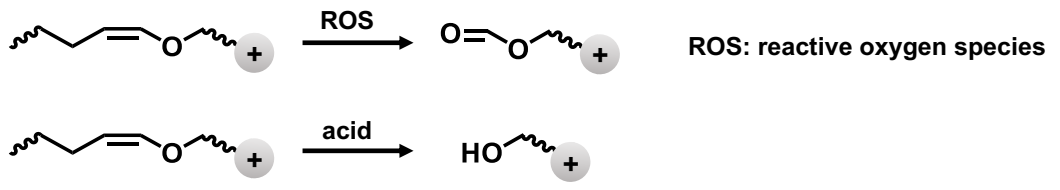

Scheme S2. Proposed pathways for the formation of side reaction products during the PB reaction. (a) Formation of the $\mathrm{CH}_{3} \bullet, \mathrm{CH}_{3} \mathrm{C}(\mathrm{O})^{\bullet}$, and $\mathrm{CH}_{3} \mathrm{C}(\mathrm{O}) \mathrm{CH}_{2} \bullet$ radicals initiated by Norrish Type I cleavage of acetone. (b) Substitution of the carbon-centered radicals to the fatty acyl chain. Other than the allylic position, substitution can happen on other sites. (c) Oxidative cleavage of the vinyl ether bond initiated by reactive oxygen species and hydrolysis of the vinyl ether chain under acidic condition. 

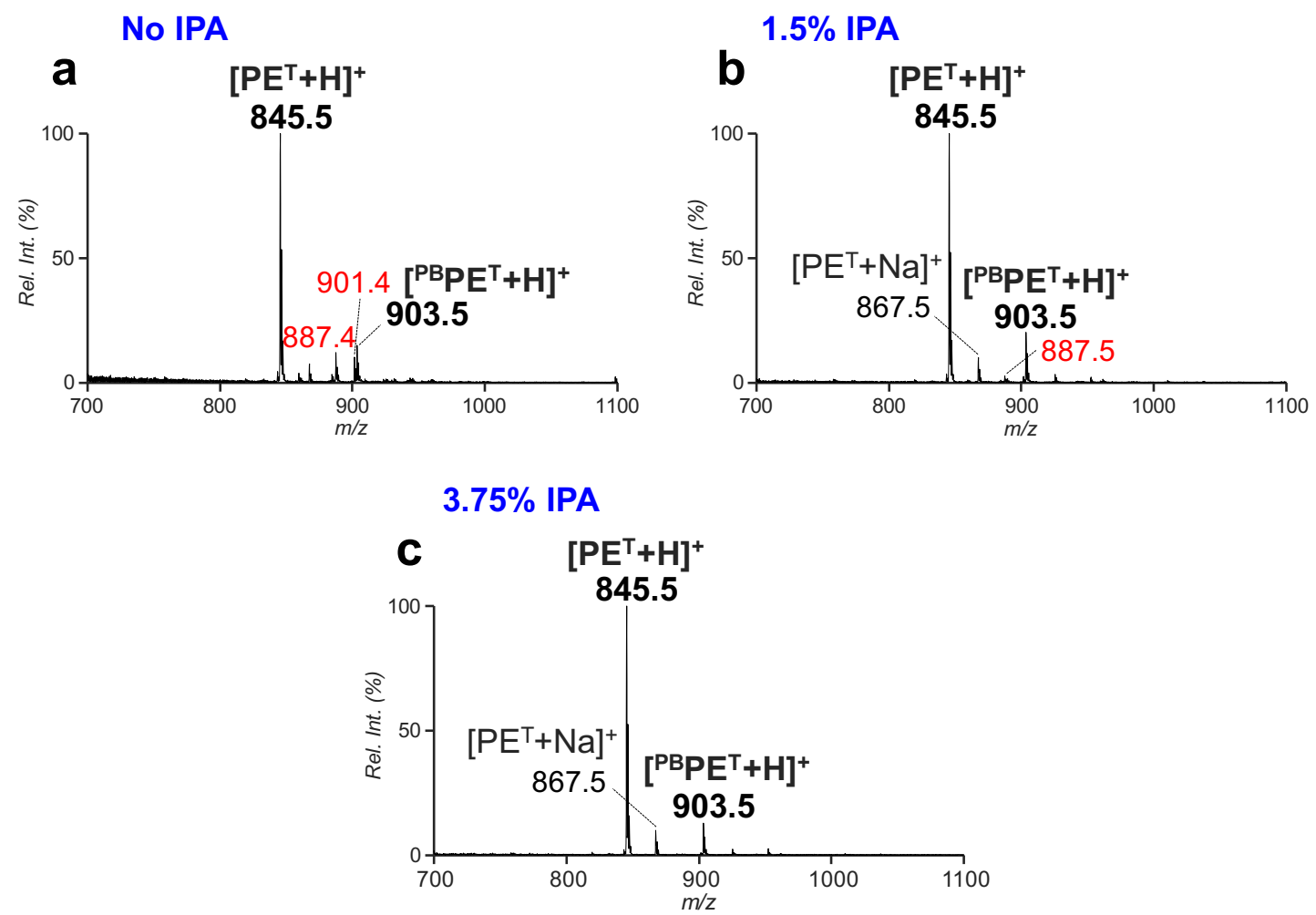

Figure S3. Positive ion HILIC-PB-MS ${ }^{1}$ spectra of $\mathrm{PE}^{\mathrm{T}}$ 16:0/18:1(9Z) using different organic mobile phases: (a) no, (b) 1.5\%, and (c) 3.75\% IPA in ACN/acetone. Peaks labeled in red are radical substitution products initiated from Norrish Type I cleavage of acetone $(\mathrm{m} / \mathrm{z} 887.4$ and $\mathrm{m} / \mathrm{z}$ 901.4). 

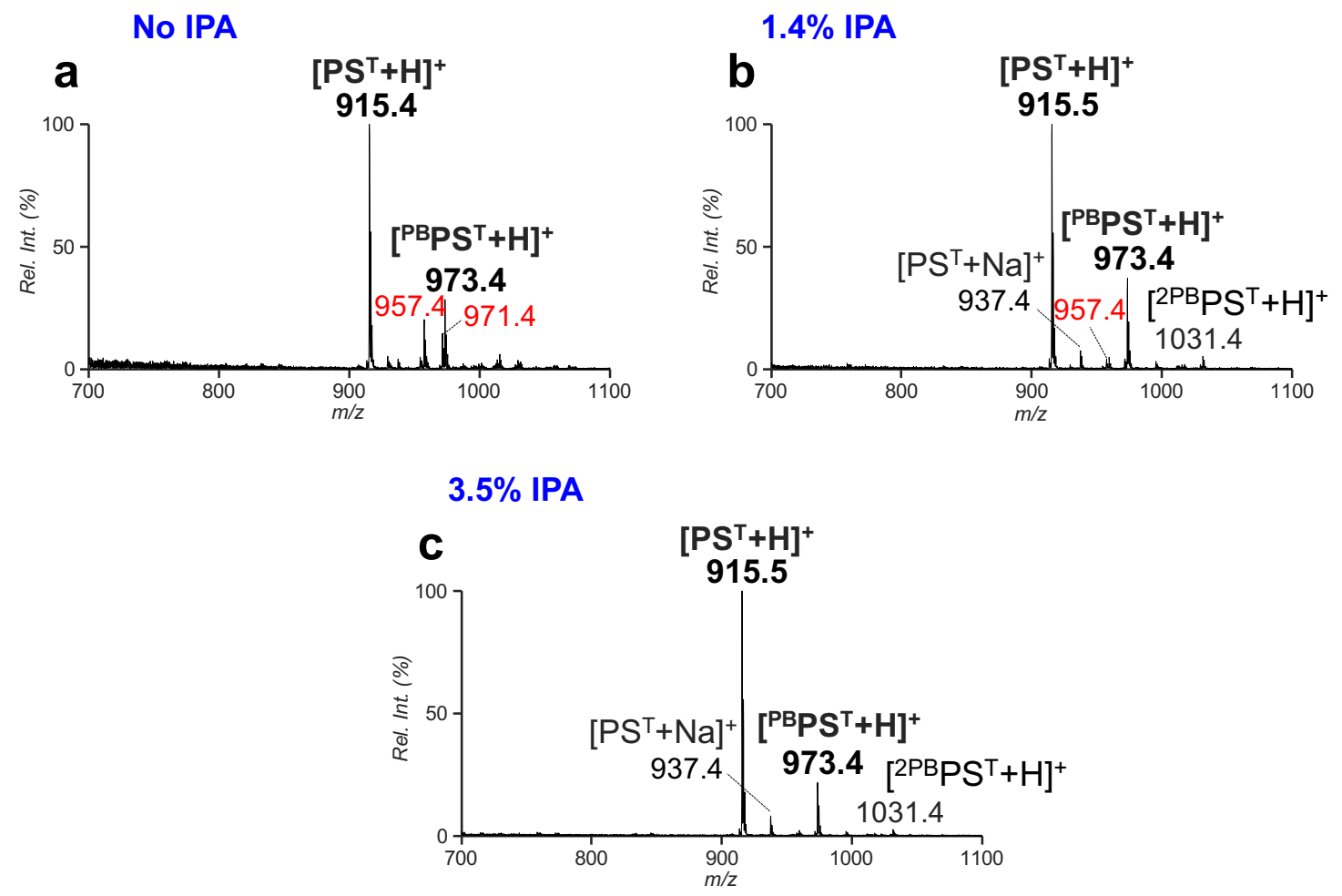

Figure S4. Positive ion HILIC-PB-MS ${ }^{1}$ spectra of $\mathrm{PS}^{\mathrm{T}} 18: 1(9 Z) / 18: 1(9 Z)$ using different organic mobile phases: (a) no, (b) 1.4\%, and (c) 3.5\% IPA in ACN/acetone. Peaks labeled in red are radical substitution products initiated from Norrish Type I cleavage of acetone $(\mathrm{m} / z$ 957.4 and $\mathrm{m} / z$ 971.4). 

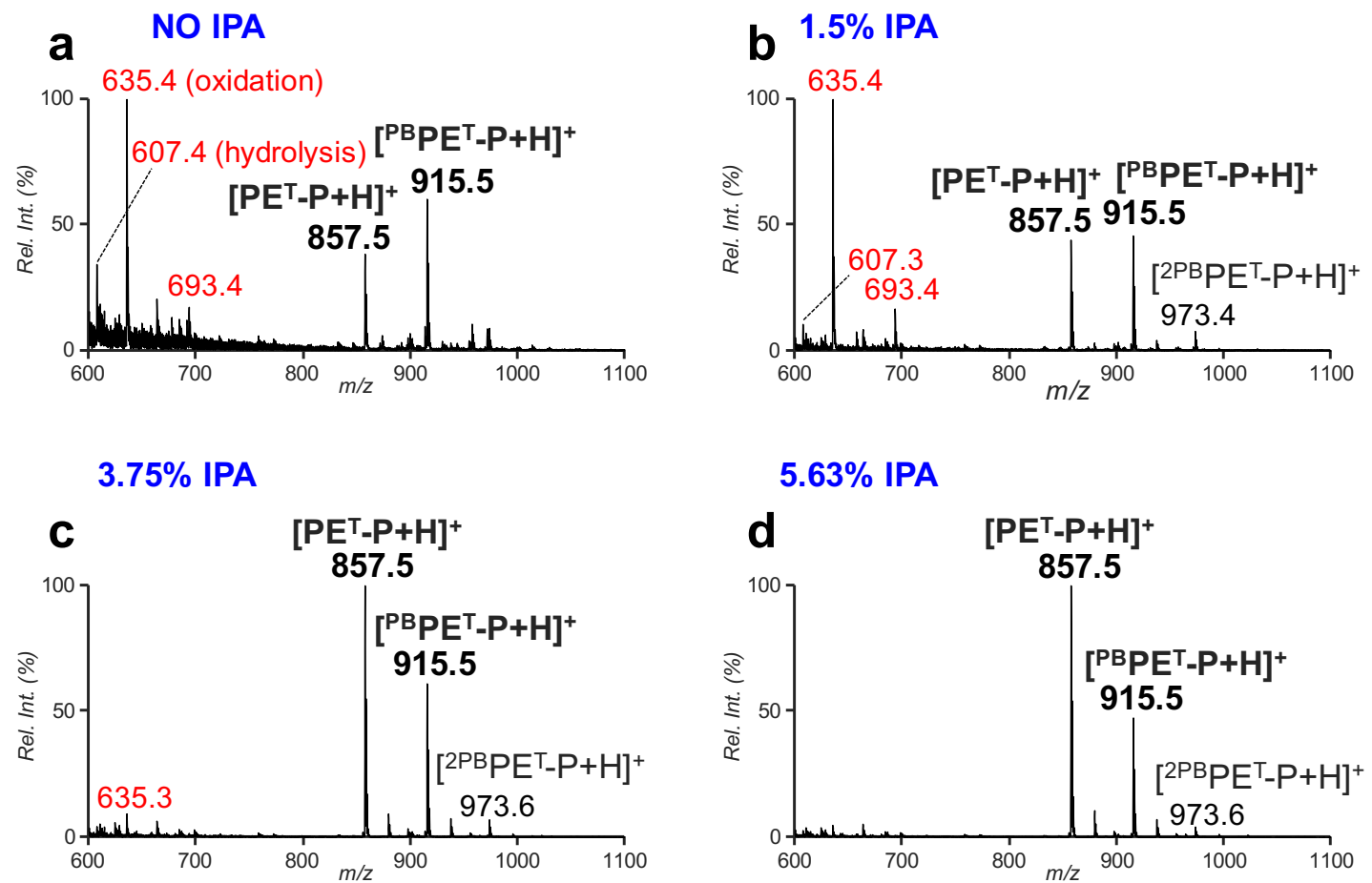

Figure S5. Positive ion HILIC-PB-MS ${ }^{1}$ spectra of $\mathrm{PE}^{\mathrm{T}} \mathrm{P}-18: 0 / 18: 1(9 Z)$ using different organic mobile phases: (a) no, (b) $1.5 \%$, (c) $3.75 \%$, and (d) $5.63 \%$ IPA in ACN/acetone. Peaks labeled in red are side reaction products due to oxidative cleavage of the vinyl ether bond $(\mathrm{m} / \mathrm{z} 635.4)$ and hydrolysis $(m / z$ 607.4). 

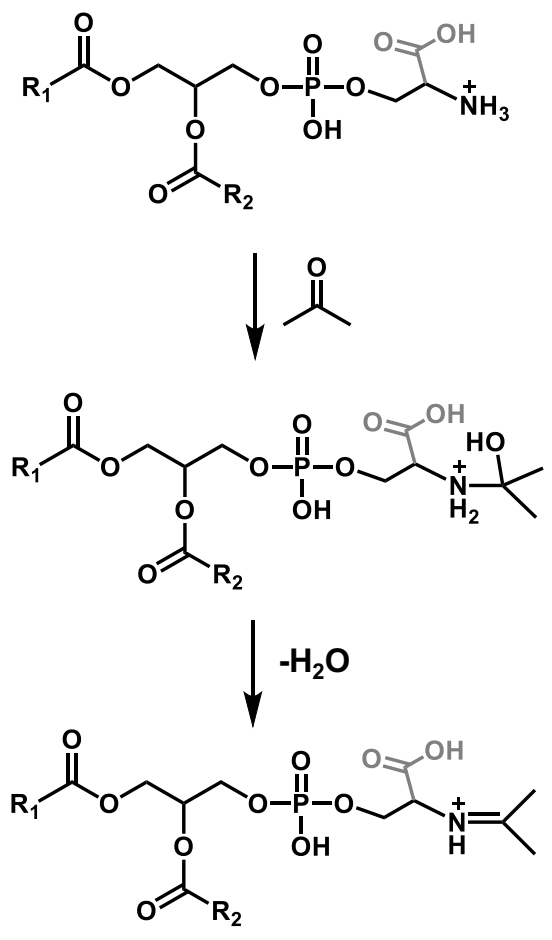

Scheme S3. Reaction scheme for Schiff base formation between aminophospholipids and acetone. 

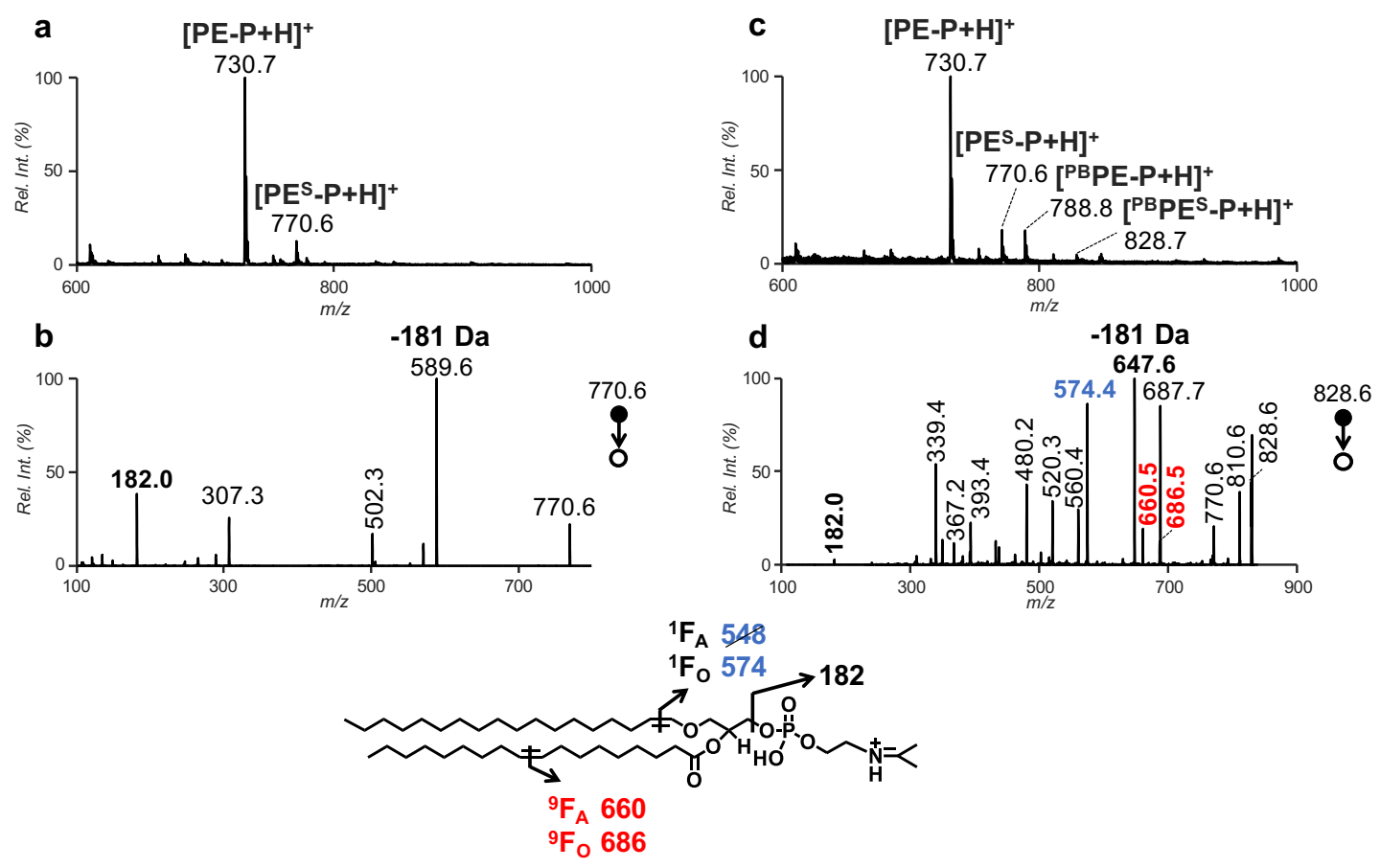

Figure S6. (a) Positive ion HILIC-MS ${ }^{1}$ spectrum of PE P-18:0/18:1(9Z). (b) HILIC-MS ${ }^{2}$ CID of Schiff base of PE P-18:0/18:1(9Z) ([PE $\left.{ }^{\mathrm{S}}-\mathrm{P}+\mathrm{H}\right]^{+}, m / z$ 770.6). (c) HILIC-PB-MS ${ }^{1}$ spectrum of PE P-18:0/18:1(9Z). (d) HILIC-PB-MS² CID of Schiff base of PE P-18:0/18:1(9Z) $\left({ }^{\mathrm{PB}} \mathrm{PE}^{\mathrm{S}}-\mathrm{P}+\mathrm{H}\right]^{+}$, $m / z$ 828.6). The superscript "S" represents Schiff base products. 


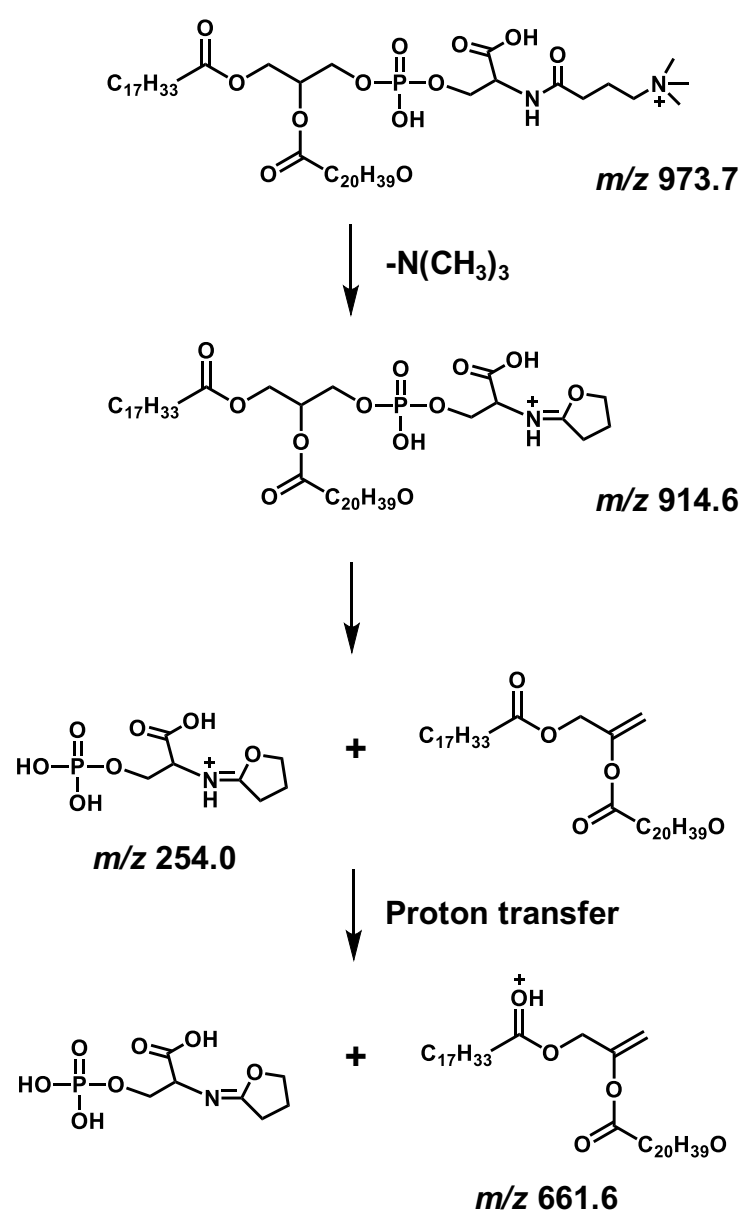

Scheme S4. Proposed fragmentation pathways for the PB products of $\mathrm{PS}^{\mathrm{T}} 18: 1(9 Z) / 18: 1(9 Z){ }^{1}$

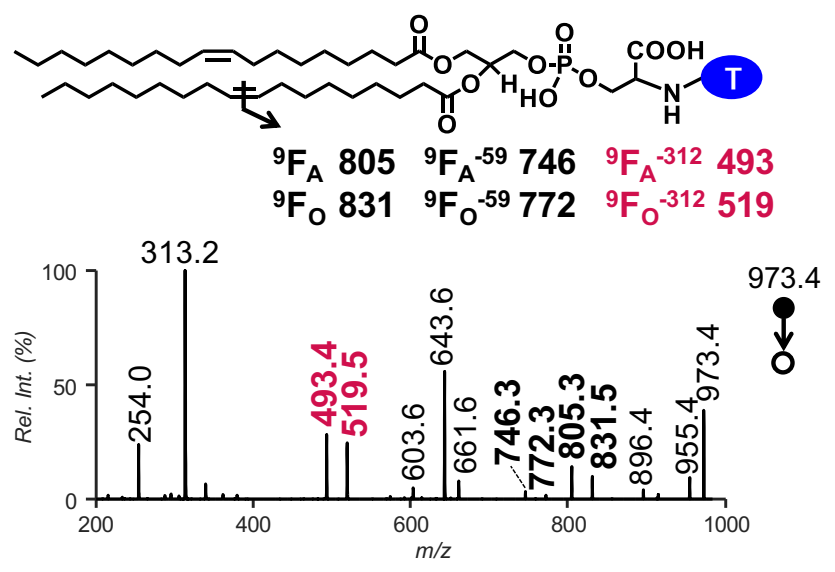

Figure S7. Positive-ion $\mathrm{MS}^{2}$ beam-type CID of the PB products of $\mathrm{PS}^{\mathrm{T}} 18: 1(9 Z) / 18: 1(9 Z)$ $\left(\left[{ }^{\mathrm{PBPS}^{\mathrm{T}}+\mathrm{H}}\right]^{+}\right.$, at $m / z$ 973.4). 


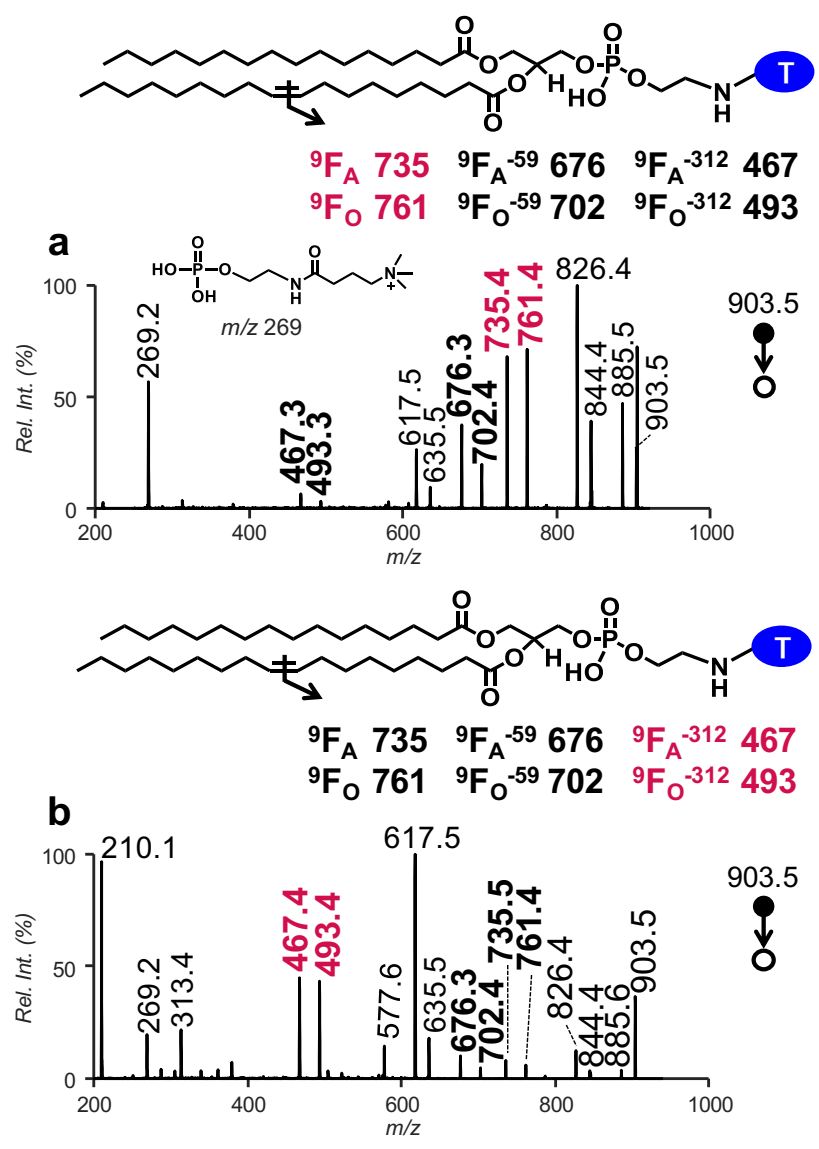

Figure S8. Positive-ion $\mathrm{MS}^{2}$ (a) ion-trap CID and (b) beam-type CID of the PB products of PE

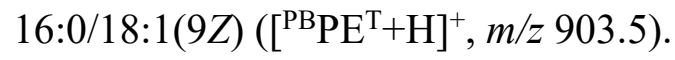

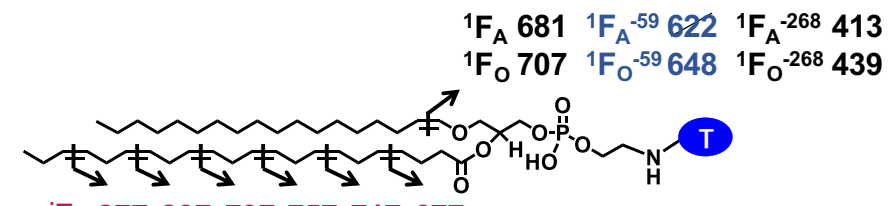

${ }^{i} F_{A} 877837797757717677$

iFo 903863823783743703

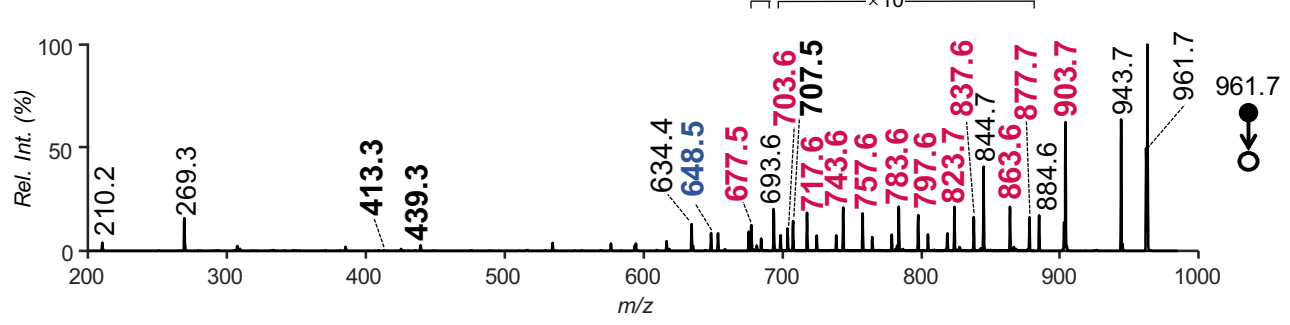

Figure S9. Positive-ion $\mathrm{MS}^{2}$ ion-trap CID of the PB products of $\mathrm{PE}^{\mathrm{T}} \mathrm{P}-18: 0 / 22: 6(4 Z, 7 Z, 10 Z, 13 Z$,

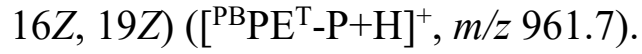


7. Analysis of Aminophospholipids from Porcine Brain Lipid Extracts

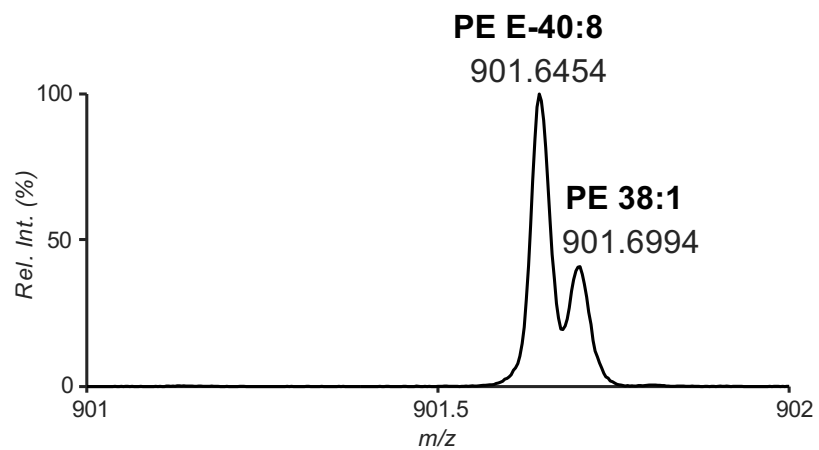

Figure S10. Differentiation of ether-PE 40:8 and PE 38:1 on a Q-TOF mass spectrometer. 

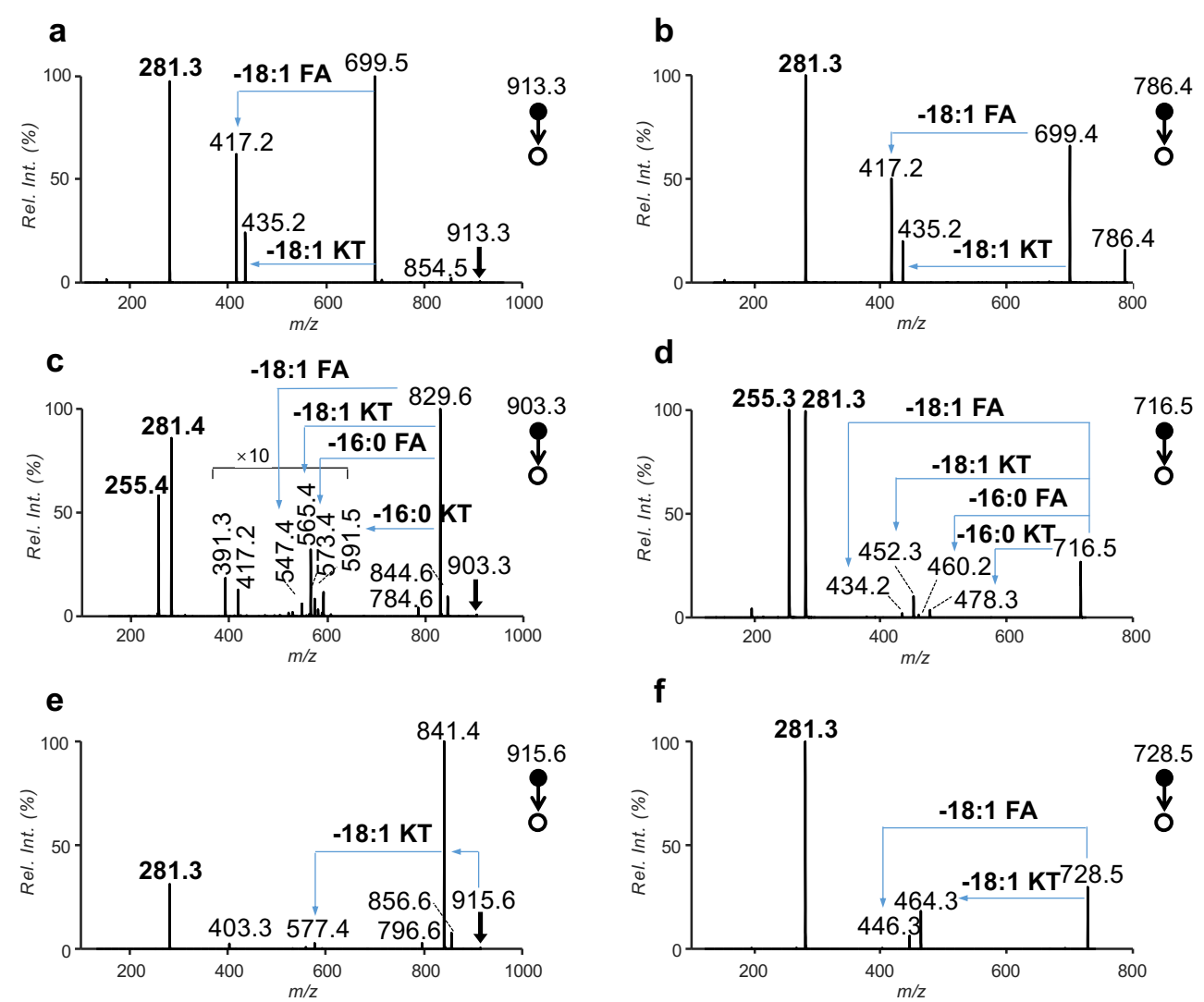

Figure S11. Negative-ion HILIC-MS² CID of deprotonated (a) PS $^{\mathrm{T}}$ 18:1(9Z)/18:1(9Z) ([PS $\left.{ }^{\mathrm{T}}-\mathrm{H}^{-}\right)$ at $m / z$ 913.3, (b) PS 18:1(9Z)/18:1(9Z) ([PS-H] $\left.]^{-}\right)$at $m / z$ 786.4, (d) PE 16:0/18:1(9Z) ([PE-H]') at $m / z$ 716.5, (f) PE P-18:0/18:1(9Z) at $m / z$ 728.5. Negative-ion HILIC-MS ${ }^{2}$ CID of acetate adducts

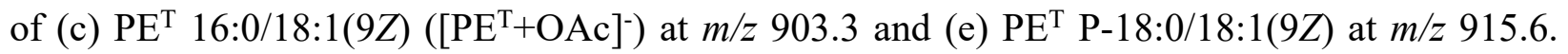
"FA" represents neutral loss of fatty acid; "KT" represents neutral loss of ketene.

CID of deprotonated PS ${ }^{\mathrm{T}}$ 18:1(9Z)/18:1(9Z) yielded an abundant phosphatidic acid anion at $\mathrm{m} / \mathrm{z}$ 699.5, as well as a less abundant fragment at $m / z 854.5$ corresponding to neutral loss of $\mathrm{N}_{(}\left(\mathrm{CH}_{3}\right)_{3}$. Also observed are carboxylate anions at $\mathrm{m} / \mathrm{z} 281.3$, representing C18:1 fatty acyl linking to the glycerol backbone, along with a pair of product ions generated via losses of fatty acyl as ketene or fatty acid from the $m / z 699.5$ ion (Figure S11a). Upon CID of acetate adducts of $\mathrm{PE}^{\mathrm{T}}$ 16:0/18:1(9Z), fatty acyl signals for $\mathrm{C} 16: 0(\mathrm{~m} / \mathrm{z} 255.4)$ and $\mathrm{C} 18: 1(\mathrm{~m} / \mathrm{z} 281.4)$, and fragments corresponding to 
loss of $\mathrm{N}\left(\mathrm{CH}_{3}\right)_{3}(\mathrm{~m} / \mathrm{z}$ 844.6) or methyl acetate $(\mathrm{m} / \mathrm{z}$ 829.6), were generated (Figure S11c). Additional product ions due to neutral losses of fatty acyls from the $m / z 829.6$ fragment were present at low abundances. For $\mathrm{PE}^{\mathrm{T}} \mathrm{P}-18: 0 / 18: 1(9 Z)$, only C18:1 carboxylate anions $(\mathrm{m} / \mathrm{z} 281.3)$ were formed (Figure S11e). Other fragmentation was similar to that of $\mathrm{PE}^{\mathrm{T}}$ 16:0/18:1(9Z).

The relative composition of $\mathrm{C}=\mathrm{C}$ location isomers was calculated from the relative abundances of the $\mathrm{C}=\mathrm{C}$ diagnostic ions with the following equation $\left(\mathrm{x}_{1}, \mathrm{x}_{2}, \mathrm{x}_{3}\right.$ represents $\mathrm{C}=\mathrm{C}$ locations):

$$
\% \operatorname{rel}_{x 1}=\frac{I_{x 1_{F}}}{I_{x 1_{F}}+I_{x 2_{F}}+I_{x 3_{F}}} \times 100 \%
$$
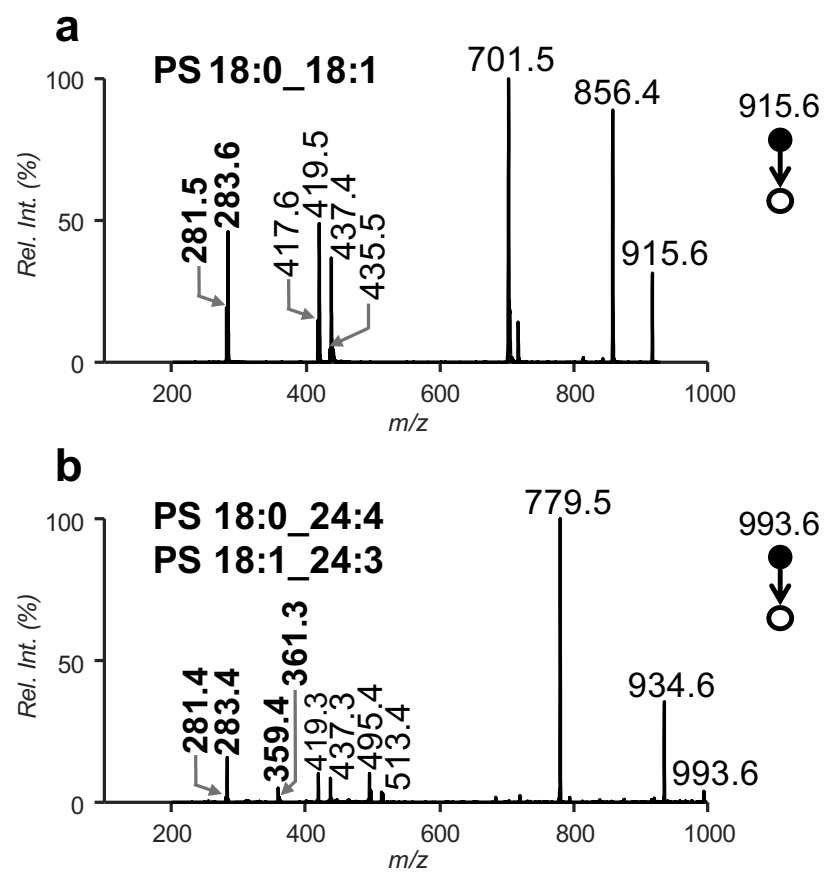

Figure S12. Negative-ion HILIC-MS ${ }^{2}$ CID of $\left[\mathrm{PS}^{\mathrm{T}}-\mathrm{H}\right]^{-}$at (a) $\mathrm{m} / z$ 915.6, (b) $\mathrm{m} / z 993.6$ from TMAB-derivatized porcine brain lipid extracts. 

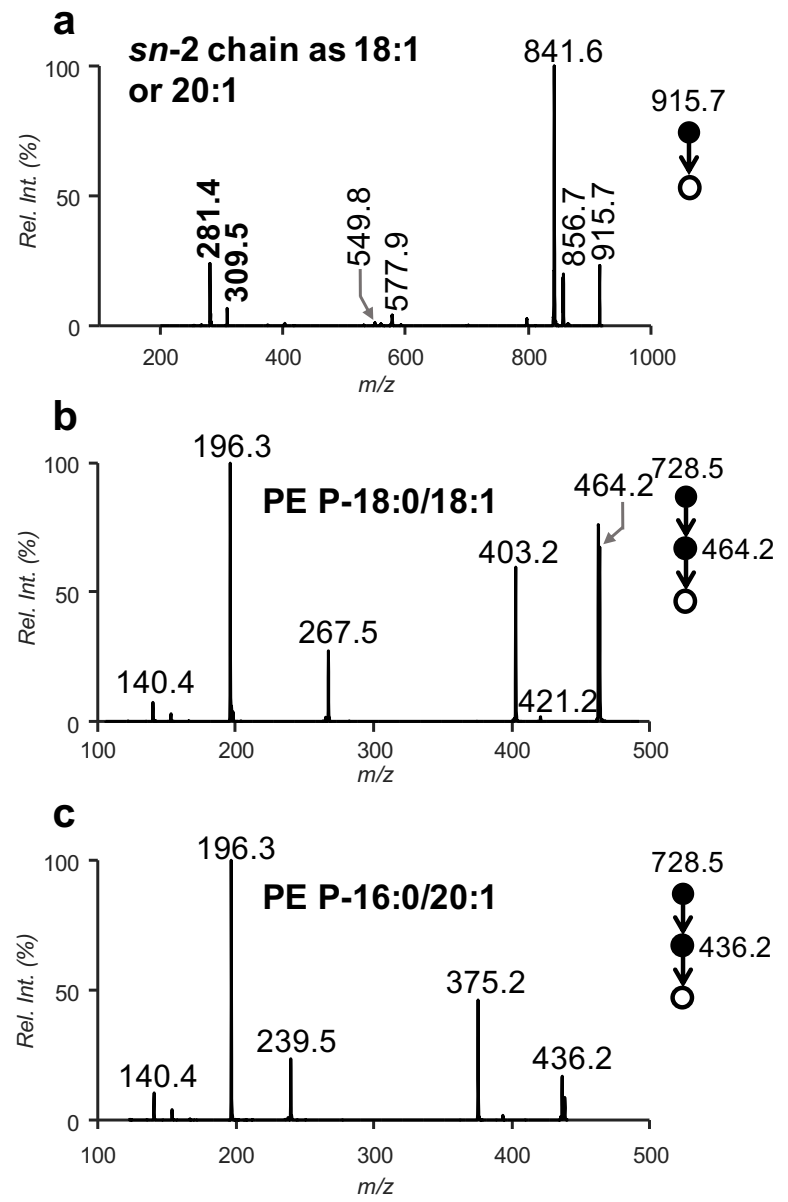

Figure S13. (a) Negative-ion HILIC-MS ${ }^{2}$ CID of acetate adducts of $\left[\mathrm{PE}^{\mathrm{T}}-\mathrm{P}+\mathrm{OAc}\right]^{-}(\mathrm{m} / z$ 915.7) from TMAB-derivatized porcine brain lipid extracts. Negative-ion HILIC-MS ${ }^{3}$ CID of (b) $m / z$ 464.2 corresponding to neutral loss of $18: 1$ ketene and (c) $m / z 436.2$ corresponding to neutral loss of 20:1 ketene from PE E-36:2 deprotonated anions at $m / z 728.5$ from porcine brain lipid extract.

$\mathrm{MS}^{3} \mathrm{CID}$ of $\mathrm{m} / \mathrm{z} 464.3$ ion generated two characteristic product ions at $\mathrm{m} / \mathrm{z} 196.3$ and 267.5, representing the loss of the $s n-1$ ether chain as a fatty alcohol $\left(\mathrm{C}_{14} \mathrm{H}_{29} \mathrm{CH}=\mathrm{CHOH}, 240 \mathrm{Da}\right)$ and the alkoxide anion $\left(\left[\mathrm{C}_{14} \mathrm{H}_{29} \mathrm{CH}=\mathrm{CH}-\mathrm{O}\right]^{-}\right)$, respectively (Figure $\left.\mathbf{S 1 3 b}\right)$. The fragmentation pattern in the $\mathrm{MS}^{3} \mathrm{CID}$ spectrum of $\mathrm{m} / \mathrm{z} 436.3$ are analogous to the above (Figure S13c). The data clarified the presence of PE P-18:0/18:1 and PE P-16:0/20:1 in this ether lipid. 


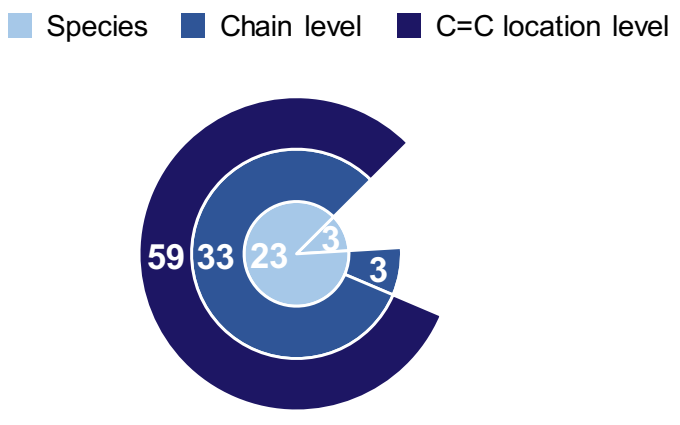

Figure S14. The number of PE identified at different structural levels.

\section{Reference}

(1) Nie, S.; Fhaner, C. J.; Liu, S.; Peake, D.; Kiyonami, R.; Huang, Y.; Reid, G. E. Characterization and multiplexed quantification of derivatized aminophospholipids. Int. J. Mass spectrom. 2015, $391,71-81$. 